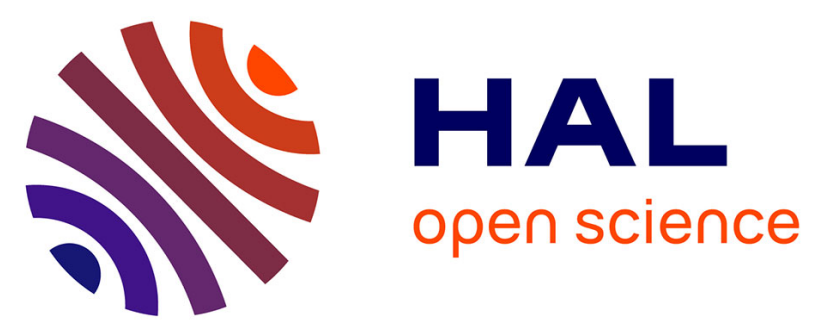

\title{
Bragg gratings written in Sn-Er-Ge codoped silica fiber: investigation of photosensitivity, thermal stability and sensing potential
}

Suchandan Pal, Tong Sun, Ken T. Grattan, Scott A. Wade, Stephen F. Collins, Gregory W. Baxter, Bernard Dussardier, Gérard Monnom

\section{To cite this version:}

Suchandan Pal, Tong Sun, Ken T. Grattan, Scott A. Wade, Stephen F. Collins, et al.. Bragg gratings written in Sn-Er-Ge codoped silica fiber: investigation of photosensitivity, thermal stability and sensing potential. Journal of the Optical Society of America. A, Optics and image science, 2004, 21 (8), pp.1503-1511. hal-00469638

\section{HAL Id: hal-00469638 https://hal.science/hal-00469638}

Submitted on 7 Apr 2010

HAL is a multi-disciplinary open access archive for the deposit and dissemination of scientific research documents, whether they are published or not. The documents may come from teaching and research institutions in France or abroad, or from public or private research centers.
L'archive ouverte pluridisciplinaire HAL, est destinée au dépôt et à la diffusion de documents scientifiques de niveau recherche, publiés ou non, émanant des établissements d'enseignement et de recherche français ou étrangers, des laboratoires publics ou privés. 


\title{
Bragg gratings written in Sn-Er-Ge-codoped silica fiber: investigation of photosensitivity, thermal stability, and sensing potential
}

\author{
Suchandan Pal \\ School of Engineering and Mathematical Sciences, City University, London EC1V 0HB, UK, and Central Electronics \\ Engineering Research Institute, Pilani, Rajasthan 333 031, India \\ Tong Sun and Kenneth T. V. Grattan \\ School of Engineering and Mathematical Sciences, City University, London EC1V 0HB, UK \\ Scott A. Wade, Stephen F. Collins, and Gregory W. Baxter \\ Optical Technology Research Laboratory, Victoria University, P.O. Box 14428, Melbourne, Victoria 8001, Australia \\ Bernard Dussardier and Gérard Monnom \\ Laboratoire de Physique de la Matière Condensée, Université de Nice-Sophia Antipolis, 06108 Nice Cedex 2,
} France

Received February 4, 2004; revised manuscript received March 30, 2004; accepted March 31, 2004

\begin{abstract}
Bragg gratings were fabricated in an $\mathrm{Sn}-\mathrm{Er}-\mathrm{Ge}-$ codoped silica fiber with a phase mask and ultraviolet radiation from a 248-nm KrF excimer laser. The photosensitivity of the fiber was examined by studying the initial growth rate of the gratings written into it. The thermal stability of the gratings was investigated and modeled in terms of both the refractive-index modulation and the effective refractive index of the fiber core. It was shown that the temperature-induced irreversible shift in the Bragg wavelength could not be predicted by the isothermal decay of the refractive-index modulation. Finally, the potential of the gratings written into the fiber is discussed in terms of their use in high-temperature-sensing applications. (๐ 2004 Optical Society of America
\end{abstract}

OCIS codes: $060.0060,060.2310,060.2370,120.0120,120.6780$.

\section{INTRODUCTION}

The numerous potential applications of UV-induced fiber Bragg gratings (FBGs) in fiber optic sensing and telecommunications ${ }^{1-3}$ have generated a significant interest in this field in recent years. However, two major factors-the photosensitivity of the fiber in which the grating is written and the thermal stability of the grating-are of prime importance in terms of choosing the most appropriate fiber to use and of the long-term functionality of the grating over a wide range of temperatures. $\mathrm{B}-\mathrm{Ge}$-codoped fiber has been reported to give a much higher level of photosensitivity ${ }^{4}$ when compared with other fibers, and the technique of hydrogen loading ${ }^{5}$ can further enhance this property of the fiber, but the gratings written in these fibers, with or without pretreatment or posttreatment, are reported to have a much poorer high-temperature stability. ${ }^{6-9}$ Alternatively, reports of work on enhanced photosensitivity in Sn-doped germanosilicate fiber ${ }^{10}$ and $\mathrm{Sn}$-silicate fiber, ${ }^{11-13}$ have been published that show improved thermal stability of the gratings. Unlike B codoping, Sn codoping does not introduce significant loss at the telecommunications window of 1.55 $\mu \mathrm{m} .{ }^{10-13}$ In addition, Sn codoping increases the mechanical strength of the fiber, ${ }^{14}$ which is very useful both in telecommunications and sensing applications, with the added advantage of enhancing the dynamic range of measurement of both strain and pressure.

In this work the photosensitivity of Sn-Er-codoped germanosilicate fiber and the thermal stability of the FBGs written into the fiber are reported; the photosensitivity of the fiber was analyzed by observing the initial growth of the gratings. The thermal stability of the FBGs was modeled, on the basis of the power law proposed by Erdogan et al. ${ }^{15}$ with an alternative approach that uses a master aging curve. ${ }^{15,16}$ This approach explains well the observed rapid thermal decay of gratings, which was seen to be followed by a very slow decay rate for any particular temperature considered. In the investigation of the thermal decay of the gratings, a blueshift observed in the Bragg wavelength ${ }^{8,17-19}$ also can be modeled by using a modified power law. At temperatures higher than ambient, the decay of the UV-induced refractive-index modulation $\left(\Delta n_{\text {mod }}\right)$ degrades the reflectivity of the grating and at the same time affects the effective refractive index ( $n_{\text {eff }}$ ) of the fiber, which is responsible for the shift of the Bragg wavelength. However, in this work different decay rates of $\Delta n_{\text {mod }}$ and $\Delta n_{\text {eff }}$ were observed in terms of the reflectivity decay and the shift of the Bragg wavelength at 
various temperatures. These decay rates are related to the complex phenomenon of the thermal decay of the UVinduced gratings, which has also been confirmed by observation of the visibility factor during the growth of a grating. This was done for various gratings, and the values of the visibility factor were also estimated before and after isothermal annealing at various selected temperatures. Both $\mathrm{Sn}^{4+}$ and $\mathrm{Er}^{3+}$ doping have been introduced in the specially fabricated fiber to enhance its properties by exploiting amplified spontaneous emission (ASE), which arises from the rare-earth doping in the fiber and, at the same time, the high-temperature sustainability of the FBGs written in the fiber that are to be used for several high-temperature-sensing applications. ${ }^{20}$

\section{EXPERIMENTAL DETAILS AND RESULTS}

\section{A. Fabrication of Fiber Bragg Gratings and the} Photosensitivity of the Special Fiber

The special Er-doped, Sn-germanosilicate fiber (core/ cladding diameter $5 / 125 \mu \mathrm{m}$, NA 0.2 , cutoff wavelength $1400 \mathrm{~nm}$ ) was designed and fabricated at the University of Nice, Nice, France, by using the modified-chemicalvapor-deposition technique. $\mathrm{Sn}, \mathrm{Er}$, and $\mathrm{Al}(1.1 \mathrm{~mol} / \mathrm{l}$, $0.025 \mathrm{~mol} / \mathrm{l}$, and $0.083 \mathrm{~mol} / \mathrm{l}$ in the solution, respectively) were introduced to achieve both high-temperature sustainability ${ }^{10-13,20}$ and a suitable fluorescence spectrum from the fiber that would cover the sensing range of the Bragg wavelength of the FBG written into it. Al was introduced to confine the $\mathrm{Er}^{3+}$ ions within the fiber core, to improve the pumping efficiency of the fiber (to generate ASE) as well as to avoid any clustering effects that may occur. $^{21}$

Initially, a type I grating was written in the specially fabricated Sn-Er-Ge-codoped, photosensitive fiber by UV light from a $\mathrm{KrF}$ excimer laser (Braggstar-500, Tuilaser $\mathrm{AG}$ ) at $248 \mathrm{~nm}$ with a pulse duration of $10 \mathrm{~ns}$ and by employing the phase mask technique (phase mask period $1061 \mathrm{~nm}$, dimensions $10 \mathrm{~mm} \times 5 \mathrm{~mm}$; zero order $2.2 \%$; Bragg Photonics Inc., Quebec, Canada). One cylindrical planoconvex lens (focal length $20 \mathrm{~cm}$ ) was used to converge the laser beam along the fiber length, and the phase mask was placed just in front of the fiber to form a light interference pattern with the +1 st order and -1 st order of the diffracted light. The grating reflectivity reached $\sim 99 \%$ within 6 min of exposure time with a laser energy of $12 \mathrm{~mJ}$ at $200 \mathrm{~Hz}$ and a pulse fluence of $\sim 180 \mathrm{~mJ} / \mathrm{cm}^{2}$, but it took a further $5 \mathrm{~min}$ to reach the highest achievable peak reflectivity of $99.6 \%$, with a comparatively slow growth rate. A redshift of the Bragg wavelength was also observed with the UV exposure during the growth of the grating, where the on-line measurement of the grating spectrum was made by using an HP-86140A (Agilent Technologies, Palo Alto, Calif.) optical spectrum analyzer. The consistency of this result was observed by fabricating a number of gratings under the same conditions.

With a reasonable assumption that the gratings were all uniform (sample tests confirmed this), the amplitude of the refractive-index modulation $\left(\Delta n_{\bmod }\right)$ was estimated by using the following equation, 1,2

$$
\Delta n_{\text {mod }}=[\lambda / \pi L n(V)] \tanh ^{-1}(R)^{1 / 2},
$$

where $\lambda$ is the operating wavelength for a grating length $L$ of reflectivity $R$ with an overlap function of the fiber $n(V)=\left\{1-\left[1 / 2.405\left(\lambda_{\text {cutoff }} / \lambda\right)\right]^{2}\right\}$. The corresponding estimated value of $\Delta n_{\text {mod }}$ was $\sim 3.3 \times 10^{-4}$ for a grating length of $\sim 6.5 \mathrm{~mm}$. Following the UV exposure during the grating fabrication, the Bragg wavelength shift was observed to be $\sim 0.82 \mathrm{~nm}$ toward the longer wavelength side, which corresponds to an increase in the effective refractive index of the fiber core $\left(\Delta n_{\text {eff }}\right)$ of $\sim 7.7 \times 10^{-4}$, this having been determined with the equation

$$
\Delta n_{\text {eff }}=\left(\Delta \lambda_{b}\right) / 2 \Lambda,
$$

where $\Delta \lambda_{b}$ is the Bragg wavelength shift during the fabrication process of the grating and $\Lambda$ is the grating period, i.e., half of the phase mask period. Figure 1 shows the variation of the amplitude of the refractive-index modulation $\left(\Delta n_{\bmod }\right)$ and the effective refractive index $\left(n_{\text {eff }}\right)$ with the UV exposure time during the growth of the grating. The evolution of the grating reflectivity and the corresponding Bragg wavelength as a function of UV exposure time are shown as an inset in Fig. 1.

To investigate the process of photosensitivity in the experiment (whether it is based on a single-photon or multiphoton process),${ }^{11,12,22}$ gratings were fabricated with different levels of laser fluence and a measurement of the growth of the gratings was carried out. If the initial growth of the refractive-index modulation $\left(\Delta n_{\text {mod }}\right)$ is assumed to be proportional to the UV exposure time $t$ with a laser fluence of $I_{p}$, then this can be expressed as

$$
\Delta n_{\bmod }=\left(I_{p}\right)^{\gamma} t
$$

and the growth rate can be represented as

$$
G=\mathrm{d} / \mathrm{d} t\left(\Delta n_{\text {mod }}\right)=\left(I_{p}\right)^{\gamma},
$$

where the numerical coefficient $\gamma$, through which the process of photosensitivity is estimated, can be determined from the slope of the growth-rate-versus-laser-fluence graph as a logarithmic plot. This is shown in Fig. 2 for this experiment. The slope of the graph was evaluated in this case to be 0.97 ( \pm 0.05 ), giving evidence that the photosensitivity of the fiber for its initial growth at the laser

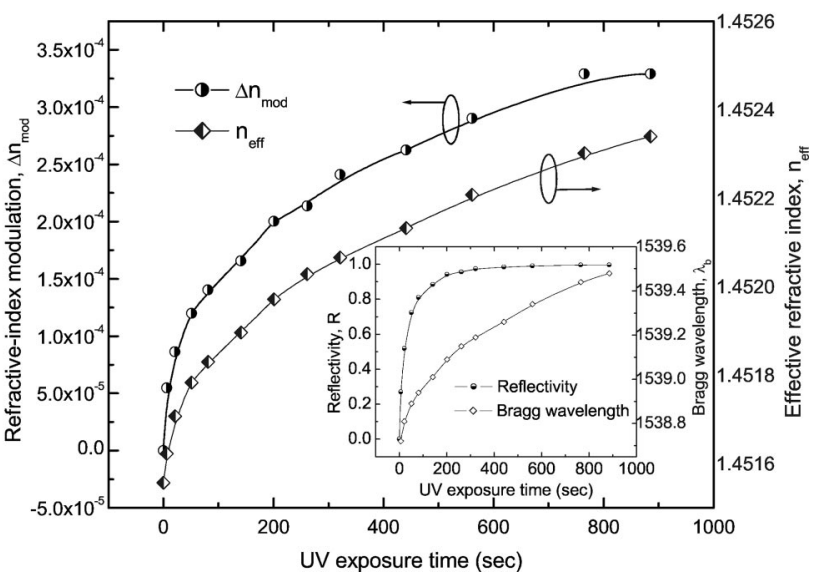

Fig. 1. Variation of the amplitude of refractive-index modulation and the effective refractive index with the UV exposure time for a laser fluence of $\sim 180 \mathrm{~mJ} / \mathrm{cm}^{2} /$ pulse. The inset shows the evolution of the grating reflectivity and the Bragg wavelength shift during the growth of the FBG. 


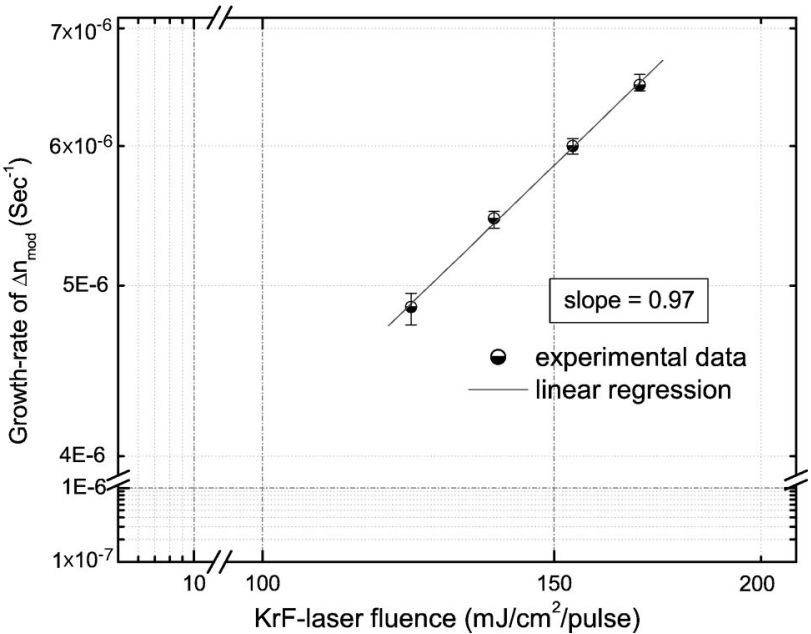

Fig. 2. Dependence of the initial grating growth rate on the laser pulse fluence. The solid line is the linear regression with a slope of 0.97 .

fluence used (at a wavelength of $248 \mathrm{~nm}$ ) is dominated by a single-photon process, although there are some reports in the literature that the total growth of the photorefractivity may arise from not only a single-photon but from a multiphoton process. ${ }^{12,22}$

\section{B. Analysis of the Thermal Stability of the Gratings}

For an analysis of thermal stability, a series of nearly identical gratings of reflectivity $\sim 90 \%$ was fabricated in the same Sn-Er-Ge-codoped fiber. Grating reflectivities were controlled by stabilizing the energy, pulse frequency, and the exposure time of the excimer laser. The estimated values of $\Delta n_{\text {mod }}$ and $\Delta n_{\text {eff }}$ [by use of Eqs. (1) and (2)] were $\sim 1.8 \times 10^{-4}$ and $\sim 4.5 \times 10^{-4}$, respectively. No saturation was required in this work because the reflectivities were measured in this study by considering only the minimum of the transmission spectrum at the Bragg wavelength, not the spectral width, which is directly related to $\Delta n_{\text {mod }}$ for strong gratings. ${ }^{16}$

The thermal degradation of the FBGs with time was monitored separately at temperatures of 100, 300, 500, 700,800 , and $900{ }^{\circ} \mathrm{C}$ by placing the individual gratings very carefully (so that there was no excess strain on the grating) in a well-calibrated CARBOLITE tube oven (Type MTF 12/38/400). Temperatures were kept constant for each measurement over a period of $\approx 8 \mathrm{~h}$, after which the gratings were returned slowly to room temperature to measure their decay in reflectivity and the irreversible blueshift of Bragg wavelength. The real-time reflectivities of the gratings were measured from the transmission spectra observed on the optical spectrum analyzer. At each temperature, a fast decay of the grating followed by a very slow decay was observed. The thermal decay was modeled in terms of the normalized integrated-coupling coefficient (NICC, $\eta$ ) because the integrated-coupling coefficient (ICC) is directly proportional to the peak reflectivity. The peak reflectivity of the grating at any time can be calculated by

$$
R=\left(1-T_{\min }\right)
$$

where $T_{\min }$ is the transmission minimum of the grating at the Bragg wavelength $\left(\lambda_{b}\right)$. The value of ICC can be calculated as

$$
\mathrm{ICC}=\tanh ^{-1}\left(R^{1 / 2}\right),
$$

which can be used subsequently for the evaluation of $\operatorname{NICC}(\eta)$ as

$$
\eta=\left[\tanh ^{-1}\left(R_{t, T}^{1 / 2}\right) / \tanh ^{-1}\left(R_{0, \mathrm{RT}}^{1 / 2}\right)\right],
$$

where $R_{t, T}$ and $R_{0, \mathrm{RT}}$ are the reflectivities after an annealing time $t$ at a temperature $T$ and the initial reflectivity at room temperature $\left(\sim 23^{\circ} \mathrm{C}\right)$, respectively.

\section{Decay in Refractive-Index Modulation ( $\left.\Delta n_{\bmod }\right)$ Based} on the Power Law

Using the experimental data obtained, we modeled the thermal decay characteristics according to the power-law function proposed by Erdogan et al. ${ }^{15}$ This can be expressed as

$$
\eta=1 /\left[1+A\left(t / t_{1}\right)^{\alpha}\right]
$$

where $t, A$, and $\alpha$ are the decay time in minutes, the power-law factor, and the power-law decay coefficient, respectively. To keep the dimensions consistent, a time, $t_{1}=1 \mathrm{~min}$, was introduced. Both $A$ and $\alpha$ are dimensionless in this form but they are temperature-dependent. It was found that the model fits the experimental data reasonably well, except for a few data at $900{ }^{\circ} \mathrm{C}$, which likely arise from the uncertainties in the measurement for very low values of reflectivities with the oscillatory pattern of the reference power spectrum. Figure 3 shows the thermal decay characteristics along with a graph fitted to the model (solid curves). The set of values of $A$ and $\alpha$ was calculated from the experimental points for each temperature according to Eq. (8). Following that, $A$ and $\alpha$ were plotted against temperature to evaluate the temperature dependence of these parameters, this being shown in Figs. 4 and 5. The variation of $\alpha$ with temperature was assumed to be linear, passing through zero on the temperature axis (K), and the temperature dependence of this parameter can be expressed as

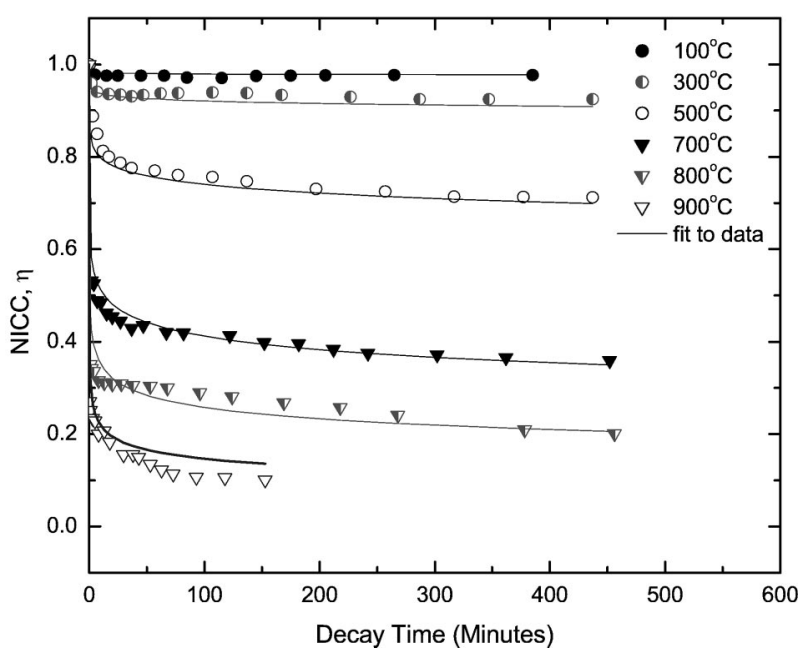

Fig. 3. Isothermal decay of the FBGs written in an Sn-Er-Gecodoped fiber with time in terms of the NICC at various temperatures. 


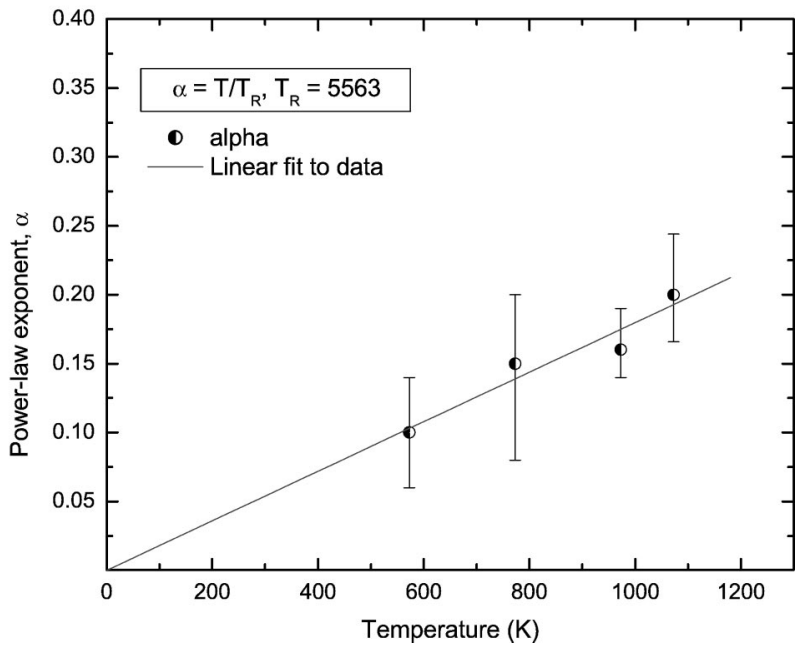

Fig. 4. Linear fit for the power-law decay coefficient $\alpha$.

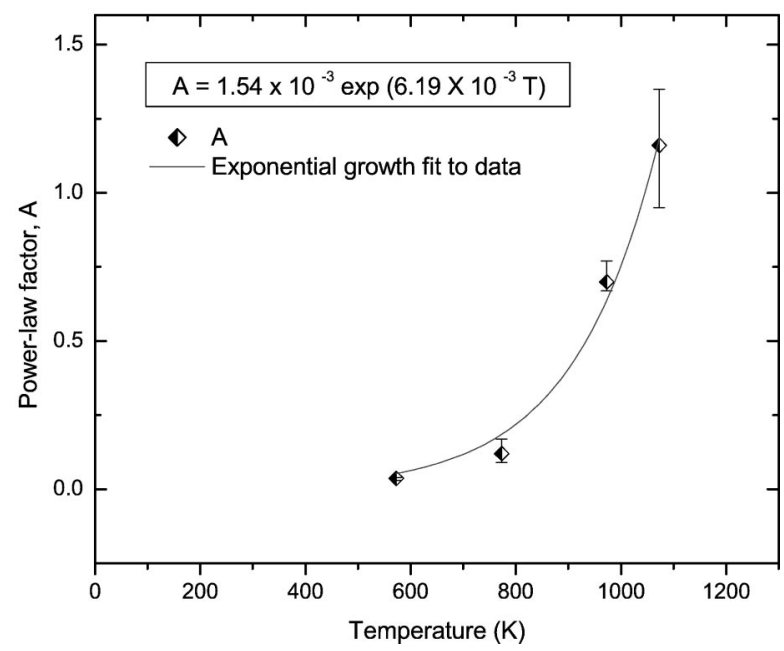

Fig. 5. Exponential fit for the power-law factor $A$.

$$
\alpha=T / T_{R},
$$

where $T$ is the temperature in Kelvin, and from the slope of the linear fit the value of $T_{R}=5563 \mathrm{~K}$ can be estimated. Figure 5 shows a plot of $A$ with temperature on a linear scale; where the value of $A$ was evaluated by exponential growth fitting, the nature of which can be written as

$$
A=A_{0} \exp (a T)
$$

with $A_{0}=1.54 \times 10^{-3}$ and $a=6.19 \times 10^{-3} \mathrm{~K}^{-1}$. The values of $T_{R}, A$, and $a$ in this work are slightly different from the brief results reported by Brambilla et al. ${ }^{13}$ for Sn-silicate fiber. The variation of the composition of the fiber and the dopants (specifically, selecting Er and Ge, in addition to $\mathrm{Sn}$ ) and the concentrations of the dopants within the core of the photosensitive fibers used are most likely to have caused these differences in the values of these parameters.
2. Decay in Refractive-Index Modulation ( $\left.\Delta n_{\bmod }\right)$ Based on the Master Aging Curve

The success in the use of the power law to represent the decay of the gratings depends on the $\alpha$-versus- $T$ and $A$-versus- $T$ relationships being expressed by Eqs. (9) and (10). These may, however, not always be correct, as a deviation of the regression may be observed from the experimental data for $\alpha$, particularly at lower temperatures. This is due mainly to the large uncertainty in the value of ICC due to the measurement errors in $T_{\min }$ for the very small grating decay at lower temperatures. Thus in this work, attempts were made to fit the data from the accelerated aging experiments at 100, 300, 500, 700, 800, and $900{ }^{\circ} \mathrm{C}$ to obtain a "master curve" by using the aging curve approach $^{8,13,15,16,23}$ to predict the operational lifetime of the grating. In this approach, it is assumed that the grating inscription causes a broad distribution of the activation energy of thermodynamically unstable traps, and the aging of any grating at any time $t$ and temperature $T$ can be described by an aging parameter ${ }^{8,13,15,16,23} E_{d}$ (the demarcation energy) by

$$
E_{d}=k_{B} T \ln (\nu t),
$$

where $k_{B}$ is Boltzmann's constant and $\nu$ is a frequency term that can be obtained from the sets of data obtained at various temperatures (say, 100, 300, 500, 700, 800, and $900^{\circ} \mathrm{C}$ ) fitted together through an iterative process. The correct shape of the master curve can also be deduced from the power-law expression ${ }^{8,15,23}$ and is described by the equation

$$
\eta\left(E_{d}\right)=1 /\left\{1+\exp \left[\left(E_{d}-\Delta E\right) / k_{B} T_{R}\right]\right\}
$$

where the total number of the trapped carriers at time $t$ was assumed to be proportional to the experimentally determined values of $\eta$. Figure 6 illustrates the master aging curve for the grating with the same (earlier) experimental data. For this plot, the optimum value of $\nu$ used was $1.54 \times 10^{13} \mathrm{~Hz}$ for the best fit to a single master curve. After the establishment of the master aging curve, the degradation of the grating for any combination of time and temperature can be projected, as discussed below.

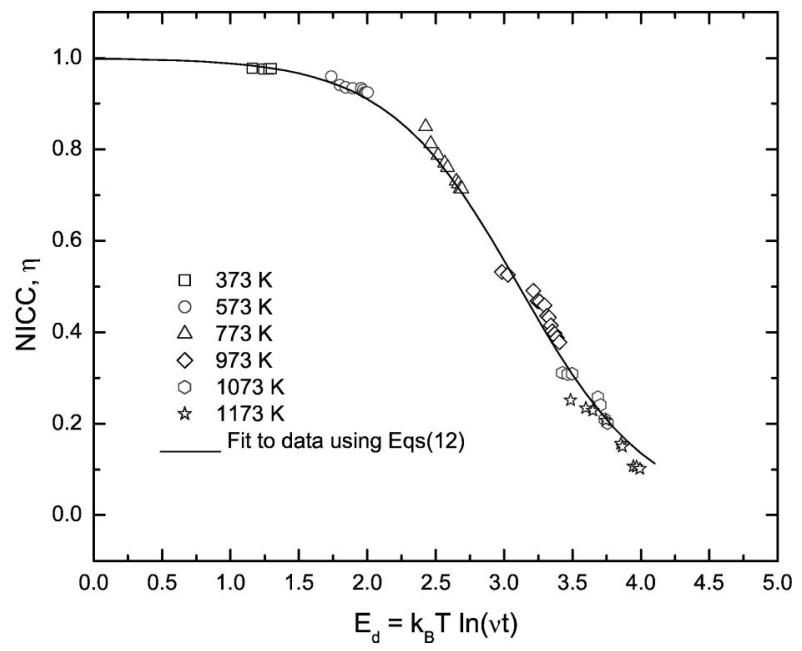

Fig. 6. NICC as a function of demarcation energy $E_{d}$ for the grating. The frequency term $(\nu)$ used for this plot is 1.54 $\times 10^{13} \mathrm{~Hz}$. 


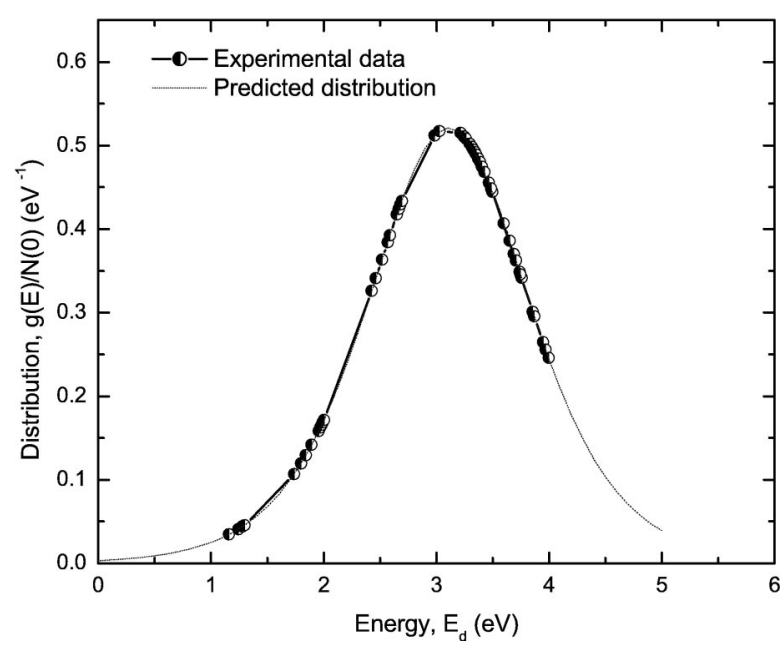

Fig. 7. Activation energy distribution plotted from the slope of Fig. 6. The symbols represent the demarcation energies actually sampled by the experiment.

The activation energy distribution for the fibers can be derived from the above coefficients, as described by Erdogan et al. ${ }^{15}$ with the peak of the bell-shaped activation energy distribution $\Delta E \approx-k_{B} T_{R} \ln \left(A_{0}\right)$ and its FWHM $\approx 3 k_{B} T_{R}$. Figure 7 represents the energy distribution of the trapped carriers for the $\mathrm{Sn}-\mathrm{Er}-\mathrm{Ge}$-codoped fiber used, as calculated from the slope of Fig. 6 . The solid portion of the curve represents the range of the demarcation energies actually sampled during the experiment, with the dotted portions the prediction arising from the model. The activation energy distribution peak was estimated at $3.1 \mathrm{eV}$ with a FWHM of $1.4 \mathrm{eV}$ in this work. Compared with the gratings fabricated in the Ge-doped fiber used by Erdogan et al. ${ }^{15}$ (distribution peak at $2.8 \mathrm{eV}$ with FWHM of $1.6 \mathrm{eV}$ ), and with gratings in B-Gecodoped fiber ${ }^{6,8}$ (distribution peak at $\sim 2.2 \mathrm{eV}$ with FWHM of $\sim 0.9 \mathrm{eV}$ ), the higher value of the activation energy in this work suggests that a higher energy is needed to "wash out" the grating, thus the grating has a higher level of sustainability at higher temperatures, although the gratings written in $\mathrm{Sn}$-silicate ${ }^{13}$ fiber have been reported more resistant to temperature, with a distribution peak at $3.7 \mathrm{eV}$ with a FWHM of $1.1 \mathrm{eV}$. Figure 8 describes a comparison of the activation energy distributions of the density of states for gratings written in B-Ge-codoped, ${ }^{8}$ Ge-doped, ${ }^{15}$ and Sn-Er-Ge-codoped fibers. The inset in the figure shows the variation of the normalized reflectivity of those gratings with temperature, taking the results as a series of isothermal steps starting from $100{ }^{\circ} \mathrm{C}$, with increments in temperature and an annealing time of $\sim 24 \mathrm{~h}$ at each step. A visual comparison shows the considerable difference in the energy distributions, which implies a significant improvement in high-temperature stability of the gratings written in $\mathrm{Sn}-$ Er-Ge-codoped fiber over those written in B-Ge- or Gedoped fibers.

\section{Decay in Effective Refractive Index $\left(n_{\text {eff }}\right)$}

The shift of the Bragg wavelength with temperature has been widely employed as the measurand in temperature sensing or in strain monitoring. In such systems, for any pre-annealed grating at each temperature studied, the Bragg wavelength shift ${ }^{8,17-19}$ is observed. So in this work, it was seen as equally important to model the decay in the effective refractive index (which is directly related to the shift of the Bragg wavelength) with time at each temperature (in the same way as the reflectivity was measured) before a grating could be used reliably in any particular application for a long time. This decay at each temperature was then modeled in a similar way, with a slight modification to the power law. The refractiveindex modulation of the fiber decreases with temperature, and this affects its effective refractive index. Thus the trend of this decay with temperature is almost identical to that of the earlier case, with the difference only in there being a lower decay rate. With this assumption, ${ }^{8}$ the power law can be modified and expressed as

$$
n_{\mathrm{eff}}(t)=n_{\mathrm{eff}}(0) /\left[1+B\left(t / t_{1}\right)^{\beta}\right]
$$

where $n_{\text {eff }}(t)$ is the effective refractive index (estimated by monitoring the Bragg wavelength) after $t$ minutes of annealing at the temperature studied, and $n_{\text {eff }}(0)$ is the initial effective refractive index at each defined and stabilized starting temperature. In the same fashion as in the power-law expression, a value of $t_{1}=1 \mathrm{~min}$ was introduced to keep the dimensions consistent. $B$ and $\beta$ may be expressed as the modified power-law factor and the modified power-law decay coefficient, respectively, for evaluating the shift in the Bragg wavelength as well as the decay in the effective refractive index. These two parameters are also dimensionless and temperature dependent, and may be calculated in the same way as was described above. Figure 9 shows the decay in effective refractive index with time at each temperature, whereas the inset in the figure describes the shift of the Bragg wavelength with time at those temperatures. The expressions for $\beta$ and $B$, through which the above equation can be fitted well to the experimental data, can be expressed as

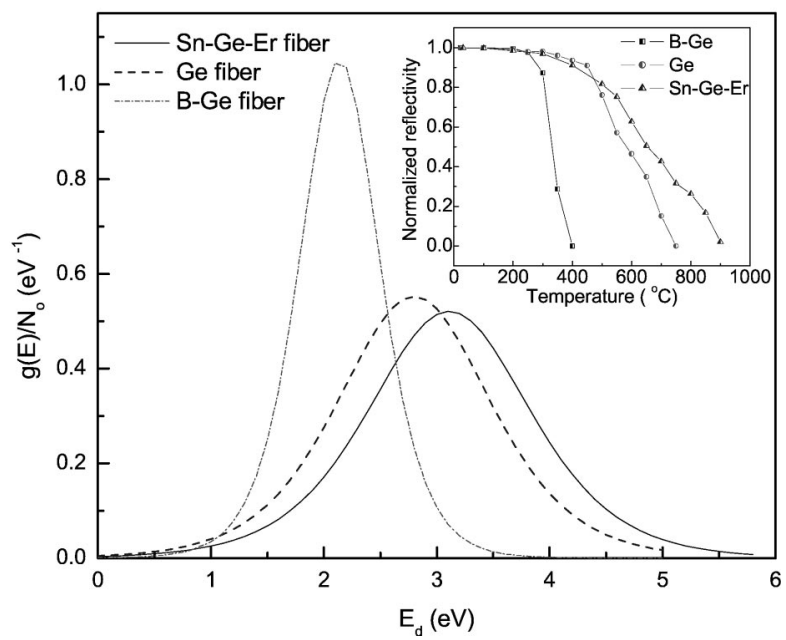

Fig. 8. Comparison of activation energy distribution of the density of states for B-Ge-(see Refs. 6 and 8), Ge-(see Ref. 15), and $\mathrm{Sn}-\mathrm{Er}-\mathrm{Ge}-\mathrm{codoped}$ fibers. The inset shows the normalized reflectivities of the gratings written in the respective fibers after $\sim 24 \mathrm{~h}$ of annealing at each temperature. 


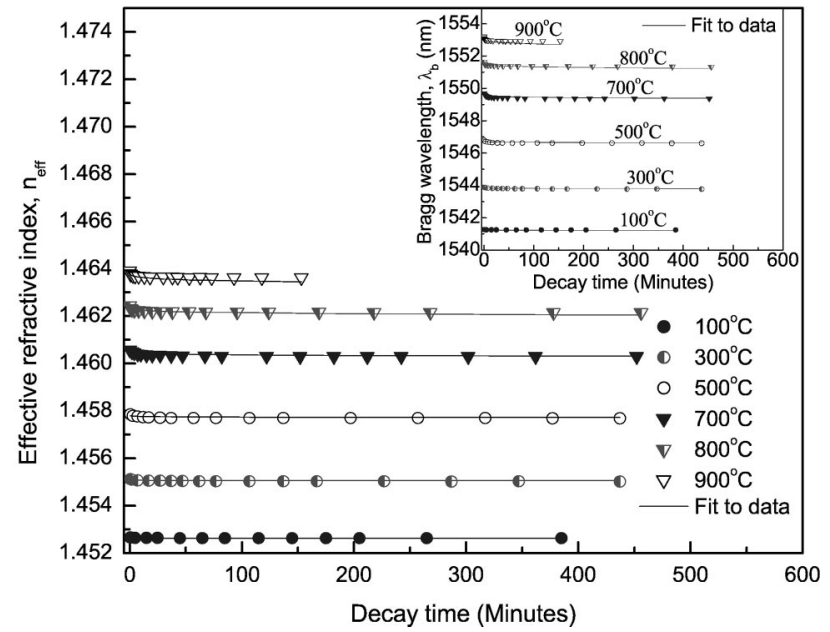

Fig. 9. Thermal degradation of the FBGs with time in terms of the effective refractive index at various temperatures. The inset shows the corresponding blueshift in the Bragg wavelengths.

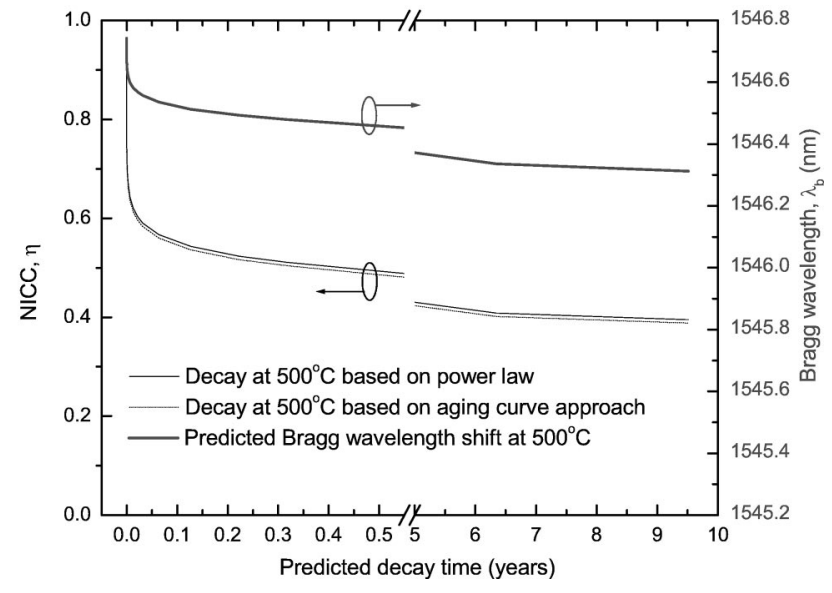

Fig. 10. Thermal response and thermal sensitivity of the gratings written in an Sn-Er-Ge-codoped fiber after proper annealing of the sample.

$$
\begin{gathered}
\beta=T / T_{\lambda} \quad \text { with } \quad T_{\lambda}=5943 \mathrm{~K}, \\
B=B_{0} \exp (b T), \quad B_{0}=5.20 \times 10^{-6}, \\
b=2.66 \times 10^{-3} \mathrm{~K}^{-1} .
\end{gathered}
$$

In Fig. 9 the modified power-law model is seen to fit well to the experimental points showing the decay in the effective refractive index, which results in the shift in the Bragg wavelength. Thus the predicted shift in the Bragg wavelength of the grating after any time, at some lower temperature, may be simulated with the above values of the parameters evaluated.

From the parameters evaluated, and by using the power law, the modified power law, and the master aging curve approach, the lifetime of the grating can be estimated in terms of the decay in the reflectivity and the shift in the Bragg wavelength. Figure 10 describes the predicted decay in reflectivity and the shift in the Bragg wavelength for a pre-annealed grating written in a $\mathrm{Sn}$ Er-Ge-codoped fiber at $500^{\circ} \mathrm{C}$ for a period of $\sim 10 \mathrm{yr}$. The results show that the NICC reaches a value of 0.66 with a shift of the Bragg wavelength of $0.18 \mathrm{~nm}$ after only one day when the grating remains at a temperature of $500{ }^{\circ} \mathrm{C}$, which is almost half of the total decay that would occur at that temperature over a predicted period of $10 \mathrm{yr}$. A negligible difference in the decay paths has been observed from the figure when the power law and the master aging curve are compared. This is mainly due to the consideration of the pre-annealed grating in this case, so that there is no truncation of the initial distribution of the activation energy. ${ }^{16}$

\section{High-Temperature-Sensing Potential}

One of the most important physical parameters for many individual applications, temperature, can be measured from a calibration of the shift of the Bragg wavelength of the grating. Sn doping in the fiber enhances the thermal stability ${ }^{10-13}$ of the grating, resulting in a wider range of measurement of temperature and establishing the prime reason for choosing a fiber with this element as one of the dopants of the core during fabrication. Temperatureinduced reversible and irreversible changes in the Bragg wavelengths $^{8,24}$ of the gratings were observed when the thermal decay of the gratings was analyzed. For sensing purposes, to enable a reproducible sensor probe to be developed, the irreversible changes of the Bragg wavelength should be eliminated to achieve repeatable measurement data by a well-defined process of annealing that stabilizes the gratings and allows only reversible changes in the Bragg wavelengths, thus resulting in a high level of repeatability over useful ranges of measurement for sensor applications.

In this work, a grating was annealed properly at $850{ }^{\circ} \mathrm{C}$ for $\sim 10 \mathrm{~h}$ before being used for temperature measurement up to $800^{\circ} \mathrm{C}$ (from room temperature in increments of $20^{\circ} \mathrm{C}$ ) by monitoring the reflection spectrum of the grating, by use of only the temperature-induced reversible changes in the Bragg wavelength. A settling time of 15 min was allowed at each temperature to ensure a thermal equilibrium between the grating and the oven before recording data for several cycles of both ascending and the descending temperatures. The measured data for the ascending and the descending temperature cycles, in terms of the Bragg wavelength change with temperature, were observed to be quite consistent over a number of repeated measurements, with a wavelength uncertainty of $\pm 0.01 \mathrm{~nm}$ (the peak-dip resolution of the optical spectrum analyzer used). The variation of the Bragg wavelength with temperature was found to be slightly nonlinear, and the temperature-dependent thermal sensitivity values of the grating were estimated to be $10.6 \mathrm{pm} /{ }^{\circ} \mathrm{C}$ and $16.8 \mathrm{pm} /{ }^{\circ} \mathrm{C}$ at room temperature $\left(\sim 23^{\circ} \mathrm{C}\right)$ and $800^{\circ} \mathrm{C}$, respectively. The thermal response of the grating, along with its temperature sensitivity, is plotted in Fig. 11.

This specially fabricated fiber has the added advantage of yielding fluorescence generated by the ASE caused by the rare-earth $\left(\mathrm{Er}^{3+}\right)$ doping. This has been shown to be very useful in the design of special fiber amplifier and fluorescence-based temperature sensing devices. ${ }^{25,26} \mathrm{Si}$ multaneous measurement of strain and temperature over a wide range of temperature can also be achieved, based on the temperature dependence of the fluorescence peak power ratio of the ASE in rare-earth-doped fiber and the 


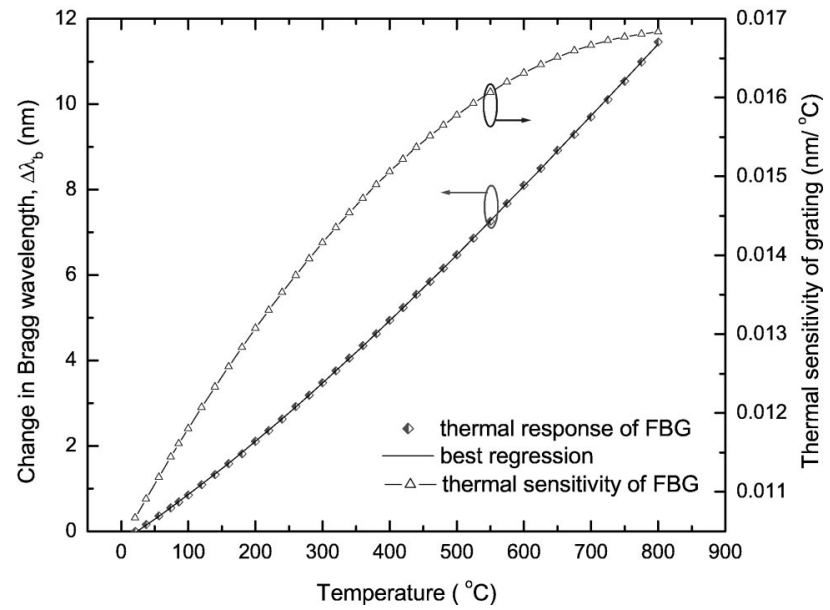

Fig. 11. Fluorescence spectra obtained at various temperatures from $\sim 10$-cm long Sn-Er-Ge-codoped fiber. The dip in the spectra indicates the grating written into the fiber.

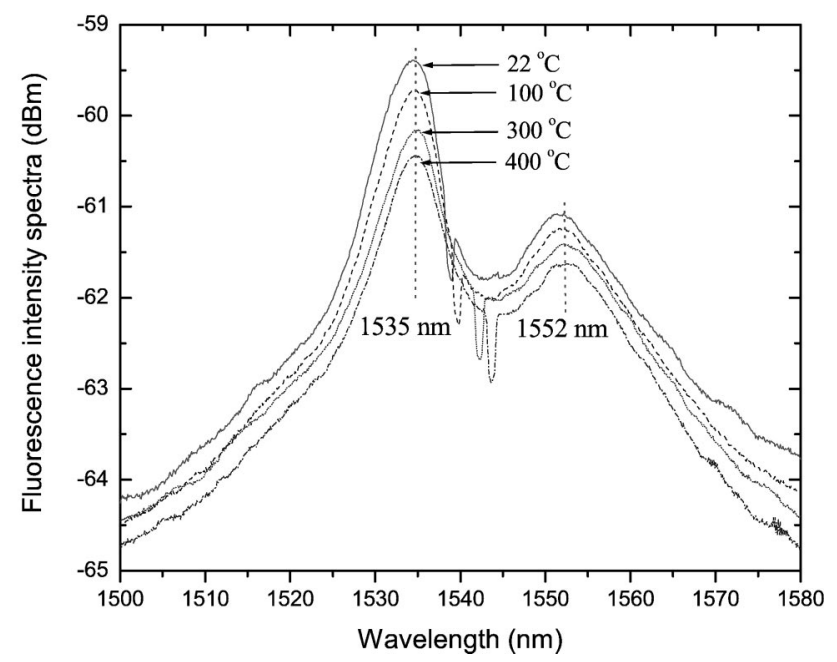

Fig. 12. Prediction of thermal decay of the grating at $500{ }^{\circ} \mathrm{C}$ according to the power law and master aging curve, along with the blueshift of the Bragg wavelength of the grating.

dual functionality of the FBG. ${ }^{20,26}$ Figure 12 shows the ASE spectra obtained from this fiber, of length $10 \mathrm{~cm}$, with a grating written in one end, where the fluorescencepeak-power-ratio approach may be applied for wavelengths of 1535 and $1552 \mathrm{~nm}$.

\section{DISCUSSION}

Although the thermal stability of the Bragg gratings written into Sn-Er-doped, germanosilicate fiber has been analyzed in this paper, both in terms of the NICC and the shift in the Bragg wavelength, both of which are directly related to the refractive-index modulation and the effective refractive index of the fiber, accurate prediction of grating stability remains difficult because of the highly complex phenomena associated with the grating decay, as discussed below.

Although the possibility of multiple peaks developing in the energy distribution has been suggested by observa- tion of a slight nonsmooth thermal decay in the grating reflectivity (see the inset of Fig. 8), only one peak has been considered in this work, to simplify the analysis of the complexity in the energy distribution arising from the presence of various dopants in the fiber (i.e., Ge, Sn, and Er) at different concentrations. An energy distribution with multiple peaks has also been reported by Rathje et $a l .{ }^{27}$ for gratings written in a H-loaded fiber. In fact, an energy distribution showing multiple peaks may be realized in this work by considering lower-temperature (below $700^{\circ} \mathrm{C}$ ) and higher-temperature (above $700^{\circ} \mathrm{C}$ ) isothermal tests separately to evaluate the decay coefficients in two different thermal states, and combining the respective energy distributions.

Second, the temperature-induced reversible changes in the grating reflectivity, 8,24 where very accurate measurement is required, has not been considered in this work because of the limited uncertainty in the measurement of the reference power level during reflectivity monitoring of the grating. The oscillatory pattern of the reference power level was possibly caused by the mismatch of the refractive index between the $\mathrm{Sn}-\mathrm{Er}-\mathrm{Ge}-$ codoped fiber used and the fibers in the connectors to the light source and the detector, as well as by the interference between the core and cladding modes that occurs in the fiber section within the splice intersections ${ }^{27}$ for the measurement of the reflectivity. The above-mentioned effect will cause little further degradation of the gratings, consideration of which has not been included here, although it has been discussed previously for gratings in B-Ge-codoped fiber by some of the authors. ${ }^{8}$

The third limitation is caused by the step-stress aging effect, ${ }^{8,28}$ which results in slightly different values of the attempted frequency $\nu$ for the master aging curve, indicating the hidden parallel degradation, which may be ruled out by use of demarcation mapping, ${ }^{28}$ although full assurance of the reliability of the accelerated testing cannot be given because of the highly complex nature of the grating decay mechanism.

Finally, although the temperature-induced reversible and irreversible changes in the Bragg wavelength shift were observed for any pre-annealed grating (although, for the chemical composition gratings, ${ }^{29}$ the irreversible changes in the Bragg wavelength have not been observed as the grating passed through the first stage of annealing during the high-temperature treatment that occurred at the time of its formation), only the irreversible changes have been considered in this study of the decay of the effective refractive index with temperature by taking the Bragg wavelength at the beginning of the experiment (time $t=0+$ ) as the reference. To separate the irreversible from the reversible shift of the Bragg wavelength, the Bragg wavelength at room temperature (say, $23^{\circ} \mathrm{C}$ ) was measured initially as $\lambda_{\mathrm{RT}}$ before annealing. If the Bragg wavelengths after annealing at any temperature and at the room temperature to which the grating returned were $\lambda_{\text {Final }}$ and $\lambda_{\text {Return }}$, respectively, then $\left(\lambda_{\text {Final }}-\lambda_{\text {Return }}\right)$ can be considered the reversible shift whereas $\left(\lambda_{\mathrm{RT}}\right.$ $\left.-\lambda_{\text {Return }}\right)$ is the irreversible shift of the Bragg wavelength. In fact, this type of analysis has also been considered in the calculation of the decay in the effective refractive index $\left(\Delta n_{\text {eff }}\right)$ of the fiber to evaluate the fringe 


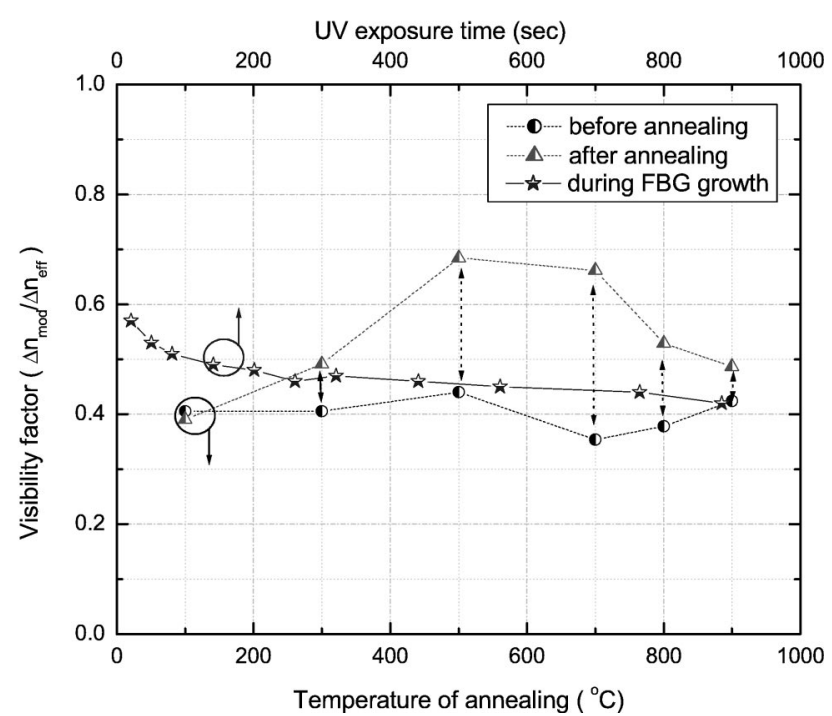

Fig. 13. Variation of the visibility factor $\left(\Delta n_{\text {mod }} / \Delta n_{\text {eff }}\right)$ of the gratings before and after annealing at various temperatures and the variation of visibility factor with time during the growth of the grating.

contrast or the "visibility factor" ( $\left.\Delta n_{\text {mod }} / \Delta n_{\text {eff }}\right)$ of the grating after each temperature cycle in this work. This is very important for the analysis of the grating decay in terms of both the reflectivity and the Bragg wavelength shift. Wang et al. ${ }^{18}$ have discussed the possible effects that may arise, taking into account the assumptions suggested by Kannan et al. ${ }^{16}$ or the variable-reactionpathway approach by Poumellec. ${ }^{30}$ Two possibilities have been predicted. The first is the case when the visibility factor remains constant. Even when the grating decay occurs after heat treatment, the characteristics of the grating then indicate that the same master aging curve can be used to predict the grating decay in terms of both the reflectivity and the Bragg wavelength shift. In the second case, the contrast or the visibility factor changes with the decay of the grating. Such behavior implies microscopic local mechanisms. To investigate this behavior, a set of experiments was carried out in which both the refractive-index modulation $\left[\Delta n_{\bmod }(296 \mathrm{~K})\right]$ and the change in the effective refractive index $\left[\Delta n_{\text {eff }}(296 \mathrm{~K})\right]$ were estimated at room temperature for all the gratings tested just before the start of the heat treatment and after completing the heat treatment at each temperature. The purpose here was to estimate the visibility factor in each case. Also, the same experiment was carried out at different points during the growth of a grating. Figure 13 shows the details of the experiment, from which it can be seen that the visibility factor increases with the decay of the grating (though the margins of the values are different for the gratings before and after the annealing being carried out at various temperatures) and decreases with the time of exposure during the growth of the grating. This means that the visibility factor for a grating is not constant with time during its growth nor during the decay. Thus the thermal stabilities of the refractive index at the bright and dark fringes are different, i.e., the decays of $\Delta n_{\text {mod }}$ and $\Delta n_{\text {eff }}$ are different for any combination of time and temperature, so the shift of the Bragg wave- length cannot be predicted simply from the decay in grating reflectivity. Different master curves may be used to predict the Bragg wavelength shift. The result obtained from the modified power law also supports the argument. Considering all of the above factors, the analysis of the grating decay can be thought of as comprising both local and nonlocal complexities. In the absence of a better analysis, and for simplicity, in the interest of keeping within the range of current theoretical approaches, the empirical power law or the master aging curve can be used for the approximate prediction of the grating lifetime.

Analysis of the results in this work has shown that the Sn-Er-Ge-codoped fiber shows a considerable level of photosensitivity when compared to other types of non-Hloaded photosensitive fibers, and that the thermal stability of the gratings written into this fiber is of great importance for individual uses when considering the wide applications of these gratings in sensing as well as in telecommunications. Results of research by some of the authors on strain and temperature sensing ${ }^{20,26}$ have been used in the investigation of the use of gratings in applications such as strain measurement inside furnace linings, where temperatures up to $850^{\circ} \mathrm{C}$ may be experienced. Equally, other structural monitoring applications, e.g., in aerospace or civil engineering, may require use at temperatures up to a few hundred degrees Celsius, illustrating the context for this type of research on FBG performance at elevated temperatures. Considering all these important applications, this work is of considerable importance and has been carried out for the first time, to the knowledge of the authors, on this valuable fiber.

\section{CONCLUSION}

The fabrication of Bragg gratings in Sn-Er-Ge-codoped silica fiber with a phase mask and ultraviolet light from a 248-nm KrF excimer laser, the mechanism of photosensitivity involved during the initial growth of the grating, and the thermal stability of the gratings were investigated thoroughly and modeled both in terms of the refractive-index modulation and the effective refractive index. The type of fiber is of particular importance because of some special applications both in sensing and telecommunications that use the combined properties of fluorescence, photosensitivity, and thermal stablity, with the added advantage of extra mechanical strength. The photosensitivity was examined with respect to the initial growth rate of the grating. The analysis of the thermal stability properties of the grating has shown that the temperature-induced irreversible shift in the Bragg wavelength could not be predicted by the decay of the refractive-index modulation. Finally, the potential of FBGs written into this fiber was discussed for hightemperature-sensing applications that use the fluorescence generated by the rare-earth doping and the dual functionality of the grating.

\section{ACKNOWLEDGMENTS}

The authors acknowledge the financial support of the UK Engineering and Physical Sciences Research Council 
(EPSRC) through various schemes and support from the Australian Research Council (ARC). S. Pal is thankful to the Commonwealth Scholarship Commission in the UK for providing a Commonwealth Scholarship and to the Central Electronics Engineering Research Institute (CEERI), Pilani, India, for the study-leave abroad.

S. Pal, the corresponding author, can be reached by e-mail at S.Pal@city.ac.uk.

\section{REFERENCES}

1. A. Othonos and K. Kalli, Fiber Bragg Gratings: Fundamentals and Applications in Telecommunications and Sensing (Artech House, Boston, Mass., 1999).

2. R. Kashyap, Fiber Bragg Gratings, Optics and Photonics Series (Academic, San Diego, Calif., 1999).

3. K. T. V. Grattan and B. T. Meggitt, eds., Optical Fiber Sensor Technology, Vol. 2 (Chapman and Hall, London, 1998).

4. D. L. Williams, B. J. Ainslie, J. R. Armitage, R. Kasyap, and R. Campbell, "Enhanced UV-photosensitivity in boron codoped germanosilicate fibers," Electron. Lett. 29, 45-47 (1993).

5. P. J. Lemaire, R. M. Atkins, V. Mizrahi, and W. A. Reed, "High pressure $\mathrm{H}_{2}$ loading as a technique for achieving ultrahigh UV photosensitivity and thermal sensitivity in $\mathrm{GeO}_{2}$-doped optical fibers," Electron. Lett. 29, 1191-1193 (1993).

6. S. R. Baker, H. N. Rourke, V. Baker, and D. Goodchild, "Thermal decay of fibre Bragg gratings written in boron and germanium codoped silica fiber," J. Lightwave Technol. 15, 1470-1477 (1997).

7. L. Dong and W. F. Liu, "Thermal decay of fiber Bragg gratings of positive and negative index changes formed at 193 $\mathrm{nm}$ in a boron co-doped germanosilicate fiber," Appl. Opt. 36, 8222-8226 (1997)

8. S. Pal, J. Mandal, T. Sun, and K. T. V. Grattan, "Analysis of thermal decay and prediction of operational lifetime for a type I boron-germanium codoped fiber Bragg grating," Appl. Opt. 42, 2188-2197 (2003).

9. I. Riant and B. Poumellec, "Thermal decay of gratings written in hydrogen-loaded germanosilicate fibers," Electron. Lett. 34, 1603-1604 (1998).

10. L. Dong, J. L. Cruz, L. Reekie, M. G. Xu, and D. N. Payne, "Enhanced photosensitivity in tin-codoped germanosilicate optical fibers," IEEE Photon. Technol. Lett. 7, 1048-1050 (1995).

11. G. Brambilla, V. Pruneri, and L. Reekie, "Photorefractive index gratings in $\mathrm{SnO}_{2}: \mathrm{SiO}_{2}$ optical fibers," Appl. Phys. Lett. 76, 807-809 (2000).

12. G. Brambilla and V. Pruneri, "Enhanced photorefractivity in tin-doped silica optical fibers (Review)," IEEE J. Sel. Top. Quantum Electron. 7, 403-408 (2001).

13. G. Brambilla and H. Rutt, "Fiber Bragg gratings with enhanced thermal stability," Appl. Phys. Lett. 80, 3259-3261 (2002).

14. K. Imamura, T. Nakai, Y. Sudo, and Y. Imada, "High reliability tin-codoped germanosilicate fibre Bragg gratingsfabricated by direct writing method," Electron. Lett. 34, 17721773 (1998)
15. T. Erdogan, V. Mizrahi, P. J. Lemaire, and D. Monoroe, "Decay of ultraviolet-induced fiber Bragg gratings," J. Appl. Phys. 76, 73-80 (1994).

16. S. Kannan, J. Z. Y. Guo, and P. J. Lemaire, "Thermal stability analysis of UV-induced fiber Bragg gratings," J. Lightwave Technol. 15, 1478-1483 (1997).

17. K. E. Chisholm, K. Sugden, and I. Bennion, "Effects of thermal annealing on Bragg fibre gratings in boron/germanium co-doped fibre," J. Phys. D 31, 61-64 (1998).

18. Q. Wang, A. Hidayat, P. Niay, and M. Douay, "Influence of blanket postexposure on the thermal stability of the spectral characteristics of gratings written in a telecommunication fiber using light at $193 \mathrm{~nm}$," J. Lightwave Technol. 18, 1078-1083 (2000)

19. T. Sun, S. Pal, J. Mandal, and K. T. V. Grattan, "Fibre Bragg grating fabrication using fluoride excimer laser for sensing and communication applications," Central Laser Facility Annual Report 2001/2002 (Central Laser Facility, Rutherford Appleton Laboratory, Oxfordshire, UK, 2002), pp. 147-149.

20. S. Pal, T. Sun, K. T. V. Grattan, S. A. Wade, S. F. Collins, G. W. Baxter, B. Dussardier, and G. Monnom, "Bragg grating performance in Er-Sn-doped germanosilicate fiber for simultaneous measurement of wide range temperature (to $500^{\circ} \mathrm{C}$ ) and strain," Rev. Sci. Instrum. 74, 4858-4862 (2003)

21. M. J. F. Digonnet, Rare-Earth-Doped Fiber Lasers and Amplifiers (Marcel Dekker, New York, 1993).

22. J. Albert, B. Malo, K. O. Hill, F. Bilodeau, D. C. Jackson, and S. Theriault, "Comparison of one-photon and twophoton effects in the photosensitivity of germanium-doped silica optical fibers exposed to intense ArF excimer laser pulses," Appl. Phys. Lett. 67, 3529-3531 (1995).

23. D. Razafimahatratra, P. Niay, M. Douay, B. Poumellec, and I. Riant, "Comparison of isochronal and isothermal decays of Bragg gratings written through continuous-wave exposure of an unloaded germanosilicate fiber," Appl. Opt. 39, 1924-1933 (2000).

24. A. Hidayat, Q. Wang, P. Niay, M. Douay, B. Poumellec, and I. Riant, "Temperature-induced reversible changes in the spectral characteristics of fiber Bragg gratings," Appl. Opt. 40, 2632-2641 (2002).

25. Y. Imai and T. Hokazono, "Fluorescence-based temperature sensing using erbium-doped optical fibers with $1.48 \mu \mathrm{m}$ pumping," Opt. Rev. 4, 117-120 (1997).

26. S. A. Wade, D. I. Forsyth, Q. Guofu, and K. T. V. Grattan, "Fiber optic sensor for dual measurement of temperature and strain using a combined fluorescent lifetime decay and fiber Bragg grating technique," Rev. Sci. Instrum. 72, 3186 3190 (2001).

27. J. Rathje, M. Kristensen, and J. E. Pedersen, "Continuous anneal method for characterizing the thermal stability of ultraviolet Bragg gratings," J. Appl. Phys. 88, 1050-1055 (2000).

28. M. J. LuValle, L. R. Copeland, S. Kannan, J. B. Judkins, and P. J. Lemaire, "A strategy for extrapolation in accelerated testing," Bell Labs Tech. J., July-September 1998, pp. 139-147.

29. M. Fokine, "Formation of thermally stable chemical composition gratings in optical fibers," J. Opt. Soc. Am. B 19, 1759-1765 (2002)

30. B. Poumellec, "Links between writing and erasure (or stability) of Bragg gratings in disordered media," J. Non-Cryst. Solids 239, 108-115 (1998). 